\title{
Stress-contingent changes in Candida albicans SAPK pathway architecture and regulation.
}

\section{Alison M. Day ${ }^{1}$, Deborah A. Smith ${ }^{1}$, Carmen M. Herrero-de-Dios²,}

Alistair J. P. Brown ${ }^{2}$ and Jan Quinn ${ }^{1}$
${ }^{1}$ Institute for Cell and Molecular Biosciences, Faculty of Medical Sciences, Newcastle University, Newcastle upon Tyne, NE2 4HH, UK ${ }^{2}$ Aberdeen Fungal Group, Institute of Medical Sciences, University of Aberdeen, Aberdeen, AB25 2ZD, UK

\section{Introduction}

-The Hog1 stress-activated protein kinase (SAPK) in Candida albicans is activated in response to divergent stresses and is essential for virulence.

-In Saccharomyces cerevisiae activation of Hog1 is controlled by a twocomponent system, composed of SIn1, Ypd1 and Ssk1 (Fig. 1). A similar twocomponent system has been shown to regulate Hog1 in C. albicans.

•Here we reveal a two-component-independent role for Ssk1 in C. albicans (Fig. 2) and demonstrate stress-specific changes in Hog1 pathway architecture and regulation

\section{Results}

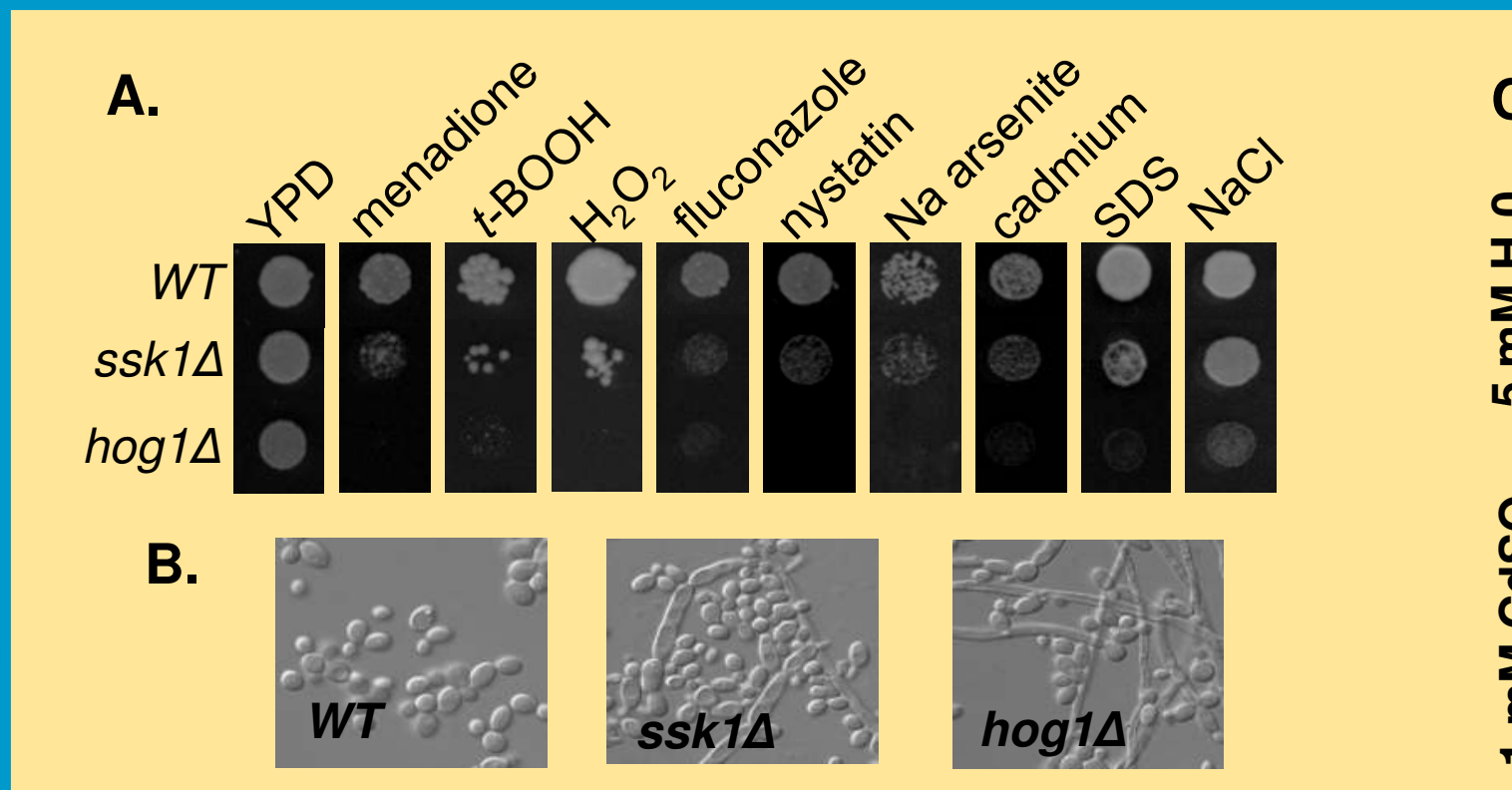

Figure 2. Ssk1 is a master regulator of the Hog1 SAPK pathway. Deletion of Ssk1 results in impaired resistance to diverse stress agents (Fig. 2A), and morphological defects (Fig. 2B), similar to that exhibited by hog14 cells. Ssk1 is required for Hog1 activation in response to diverse stress conditions (Fig. 2C).
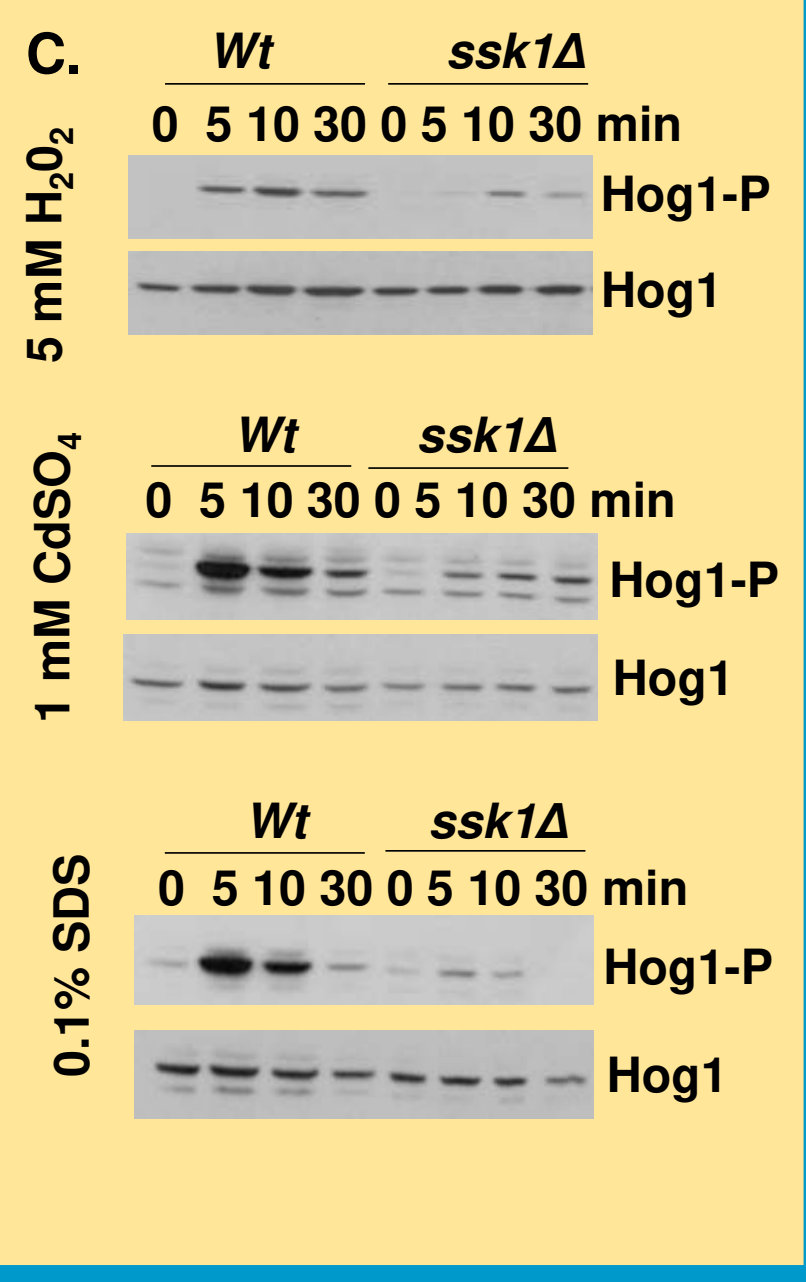

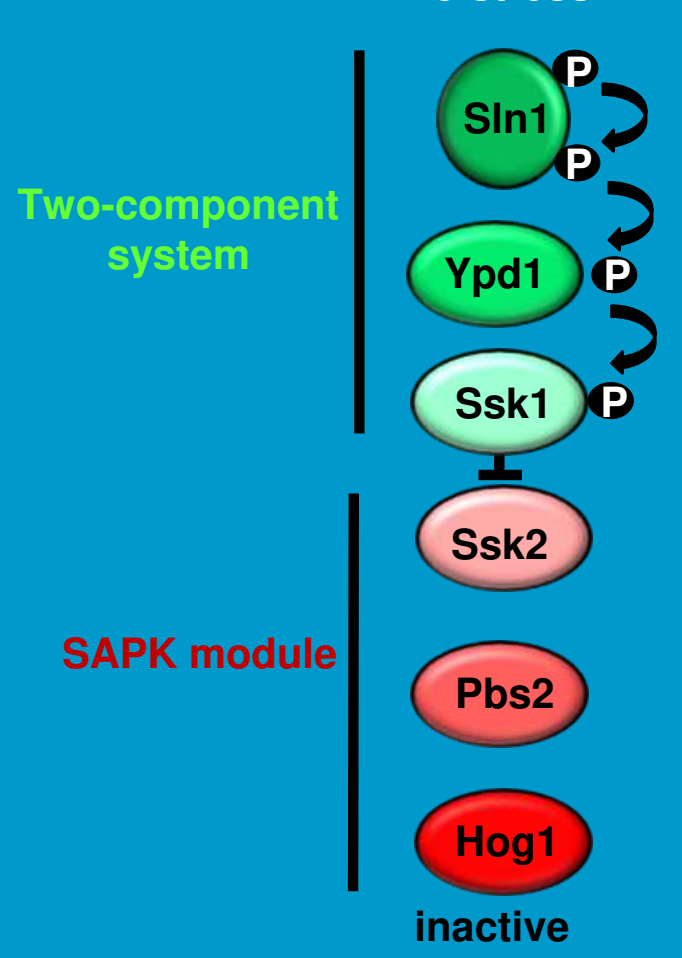

Figure 1. Two-component mediated regulation of Hog1 in S. cerevisiae.

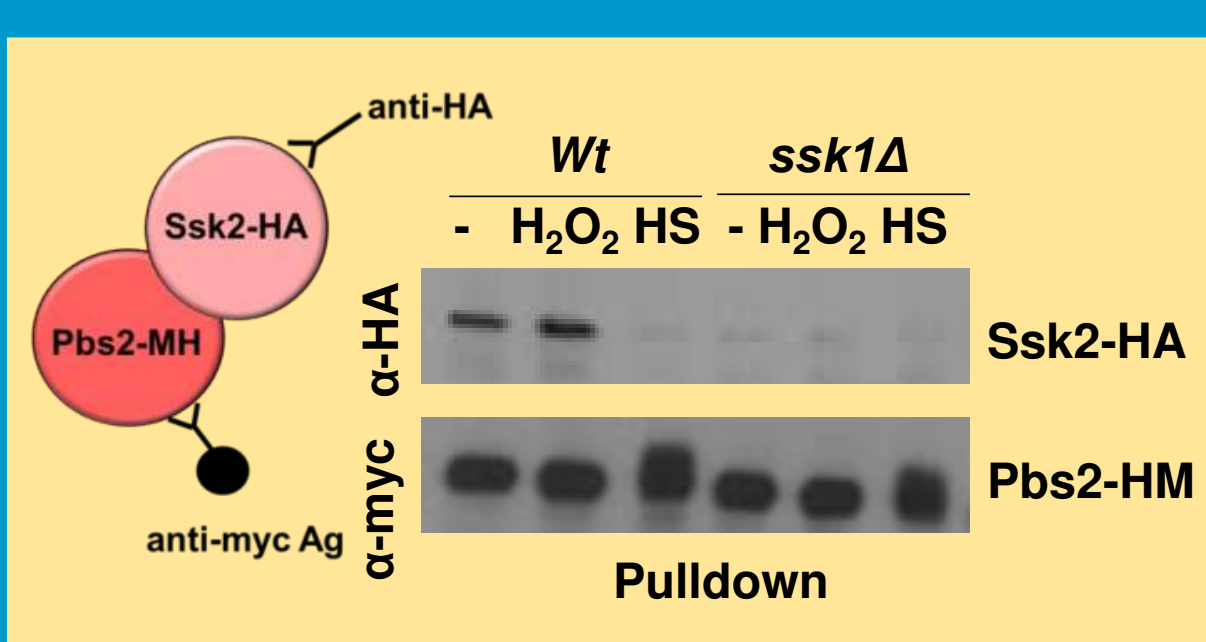

Figure 3. Ssk1 functions as a scaffold protein. Ssk1 mediates an interaction between Ssk2 and Pbs2. Co-IP experiments show that the Ssk2Pbs2 interaction is lost under conditions of high $\mathrm{NaCl}$ (HS) but not $\mathrm{H}_{2} \mathrm{O}_{2}$ stress.
A.

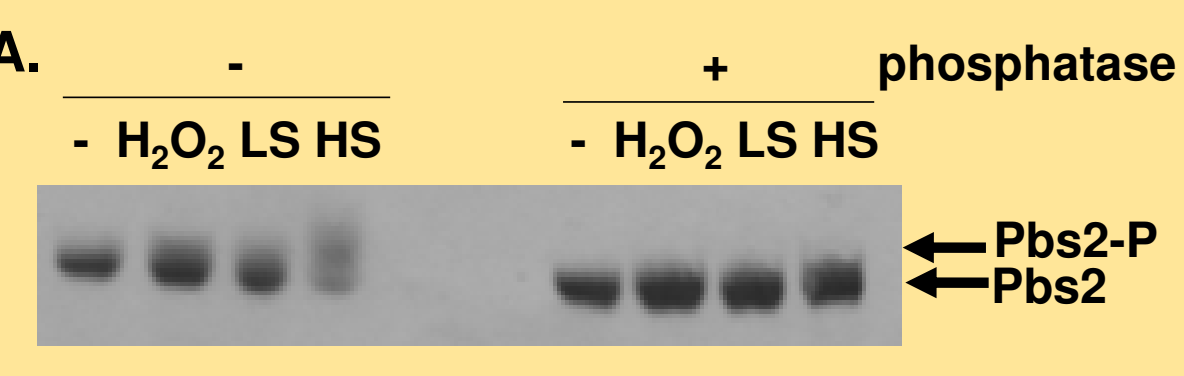

B.

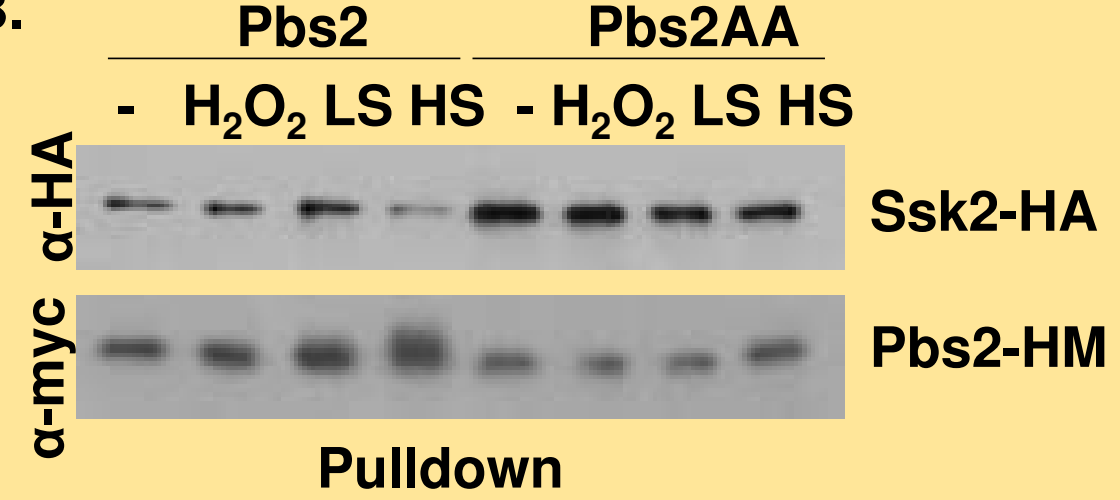

Figure 4. Phosphorylation of Pbs2 triggers its release from Ssk2. Pbs2 becomes inducibly phosphorylated following high $\mathrm{NaCl}$ (HS) stress (Fig. 4A) and this promotes its release from the Ssk2 MAPKKK (Fig. 4B). The interaction between Ssk2 and non-phosphorylatable Pbs2 $^{\text {AA }}$ is maintained (Fig. 4B).

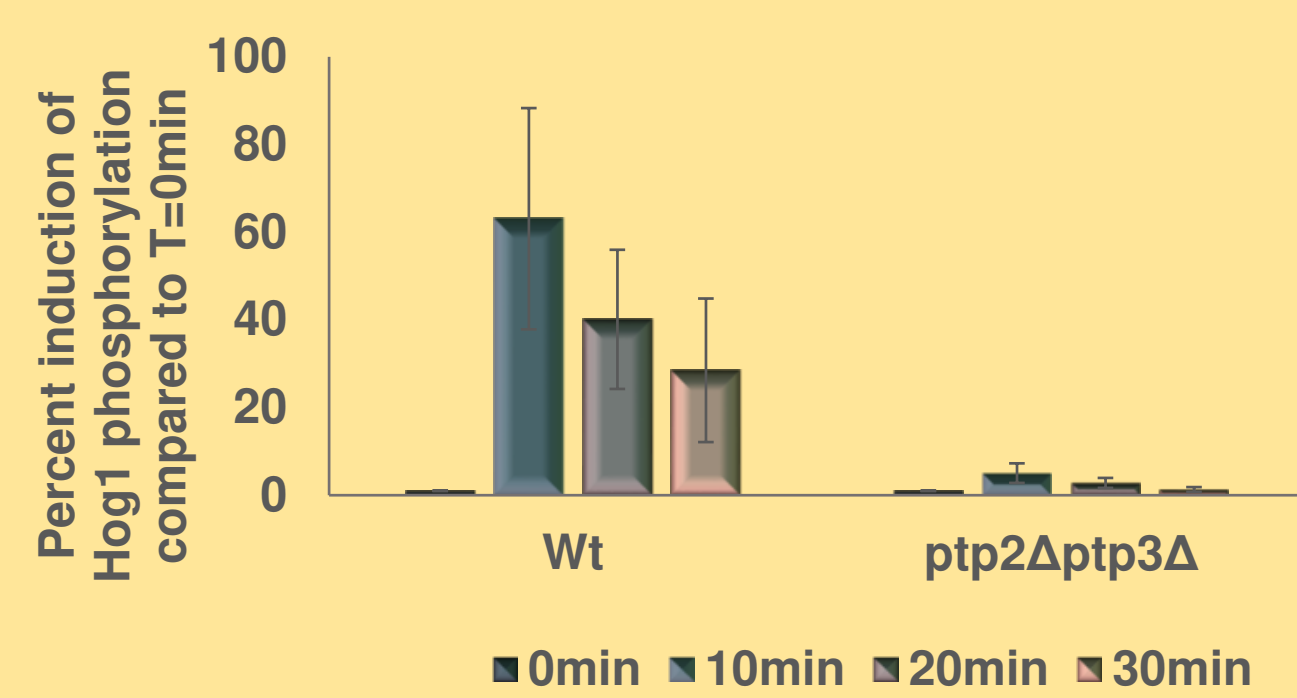

Figure 5. Hog1 phosphorylation in response to $\mathrm{H}_{2} \mathrm{O}_{2}$ is dependent on Ptp2 and Ptp3. Induction of Hog1 phosphorylation in response to $\mathrm{H}_{2} \mathrm{O}_{2}$ is

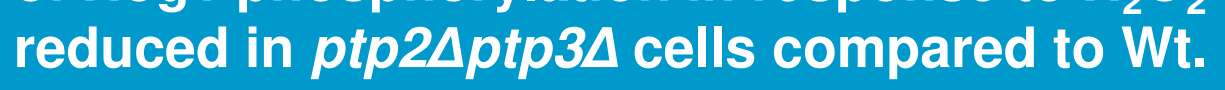

\section{Conclusions}

.Ssk1 functions as a scaffold to promote Ssk2-Pbs2 interactions within

the Hog1 SAPK module.

- This is important to promote basal levels of Hog1-P and Hog1 activation to stresses which do not signal through increased activation of Pbs2.

$\cdot \mathrm{H}_{2} \mathrm{O}_{2}$ mediated Hog1 activation requires Ssk1 scaffold-mediated upstream signalling and inactivation of the negative regulators Ptp2 and Ptp3.

-Salt mediated Hog1 activation does not require Ssk1 as this stress induces Pbs2-P which triggers Pbs2 release from the Ssk1 scaffold.

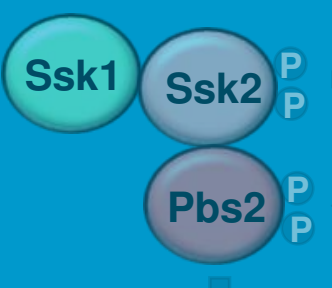

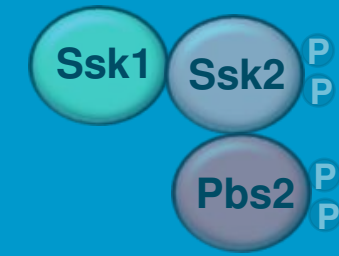

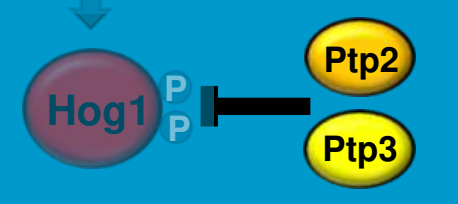

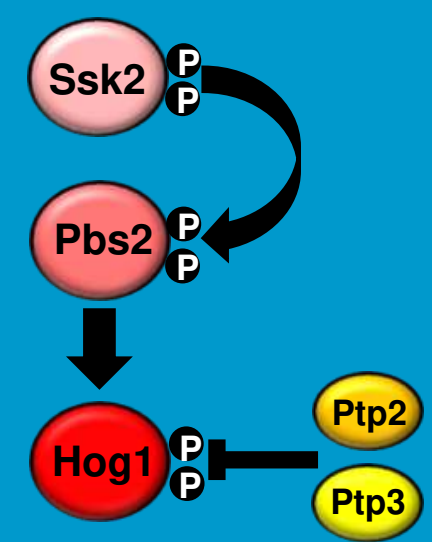

